\title{
POSTERS IN A SAMPLE OF PROFESSIONAL WORKSITES HAVE NO EFFECT ON OBJECTIVELY MEASURED PHYSICAL ACTIVITY
}

\author{
Hannah M Badland \\ Grant M Schofield
}

Centre for Physical Activity and Nutrition Research

Auckland University of Technology

\begin{abstract}
Issue addressed: Whether posters promoting stair use are effective in increasing objectively measured physical activity in a sample of New Zealand professional employees.
\end{abstract}

Methods: Forty-six participants (27 men and 19 women) recruited from two professional worksites, wore one sealed pedometer during all waking hours (total physical activity [TPV]), and another sealed pedometer during working hours (worksite physical activity [WPV]), for three days, over four separate occasions. The study protocol was a crossover design with the first worksite receiving the treatment (posters) for three weeks, followed by a sixweek wash out period, then a three-week control. The second worksite received the control prior to the treatment period. Measurements were taken at the beginning and end of each three-week block.

Results: The posters were ineffective at increasing objectively measured work and total physical activity levels in this sample. Trivial (0.04) to moderate Cohen effect sizes $(-0.79)$ were shown. When posters were visible in the worksites, mean step counts decreased (-868 steps [WPV], and - 
1861 steps [TPV]). Women's step counts (-9\% [WPV] and -13\% [TPV]) were more negatively affected by the posters appearance when compared to men $(-2 \%[\mathrm{WPV}]$ and $-8 \%[\mathrm{TPV}])$.

So what? Based on these results, the posters had little effect on physical activity for this sample. However, it was possible that an unknown confounding factor caused small decreases in step counts that disguised an otherwise positive result.

Key Words: Worksite, physical activity, pedometer Word Count: 1211 


\section{Introduction}

Physical activity is a primary measure to prevent many noncommunicable lifestyle diseases (1), and increasing evidence exists regarding the health benefits of accumulating physical activity in small bouts during the day $(2,3)$. Stair-climbing is an easy way to accumulate these small portions of physical activity throughout the day, while providing health benefits associated with being physically active $(4,5)$.

Posters used to encourage physical activity have been shown to be effective in increasing stair use in a variety of settings, including worksites (6, 7), universities $(8,9,10)$, airports (9), health care setting (11), and shopping centres $(12,13,14)$. Nevertheless, a major limitation regarding most of these studies is that the absolute number of people using the stairs were the primary evaluation measure $(6,9,10,11)$. To date, no intervention study using posters to increase physical activity has used an objective measure of physical activity within a confined sample to assess behaviour change.

\section{Methods}

\section{Study population and characteristics}

A convenience sample of participants was recruited from two Council departments in New Zealand. Forty-six (12\%) employees (27 men, 19 women) consented and completed all four testing sessions. All participants in this non-representative sample were engaged in professional, office-based duties. Twenty-one participants worked on the ground floor, with the remainder being based on the $1^{\text {st }}, 4^{\text {th }}$, and $5^{\text {th }}$ floors. The ground floor location did not inhibit inter-storey use as bathroom, cafeteria, and meeting facilities 
were located on other levels. The Auckland University of Technology Ethics Committee approved the study protocol.

Study design

A crossover design was applied to limit unknown seasonal variations that may have affected stair use and to increase the power of the small sample size (15). Worksite 1 (WS1) $(\mathrm{N}=24)$ was exposed to the poster intervention for three-weeks (measured pre and post), followed by a six-week washout period, succeeded by a three-week control period (measured pre and post). Worksite 2 (WS2) $(\mathrm{N}=22)$ had the study conducted in the reverse order. Data collection points were reported as Measures 1, 2, 3, and 4. Measure 2 was the period when posters were visible.

\section{Posters}

Two sizes of posters were used for the study. The small signs measured $14.5 \mathrm{~cm} \times 21 \mathrm{~cm}$ (A5) and the banner posters measured $67 \mathrm{~cm} \times$ $28.5 \mathrm{~cm}$. The small posters stated 'Better Steps to Health; use the stairs instead of the lift', while the large sign incorporated three messages, 'Better steps to health; use the stairs instead of the lift, take a walk during your break, drive less and walk more'. Formative qualitative analysis (focus groups) was conducted at two worksites prior to implementation (16). In the present study, 10 small posters and 8 banner posters were visible at each worksite. The small posters were mounted adjacent to elevator buttons and on bulletin boards. The banner posters appeared on stairwell landing walls only. Both sizes of posters were visible from the hallways. 


\section{Measurement}

Although pedometers do not solely count stair use, they are useful tools to determine overall changes in ambulatory movement and are suitable for detecting individual physical activity responses to the poster intervention. Participants wore two sealed hip-mounted pedometers (Yamax Digiwalker SW-700) over four periods. One pedometer (labelled work) was worn for the duration of the working day, whereas the other pedometer was worn for the entire three-day period. Pedometers were distributed for each measurement period at Monday 9:00am and collected Thursday 9:00am. Participants were not informed of the intentions of the study.

\section{Statistical Analyses}

Preliminary findings were expressed as pedometer step count changes. This was established by using the difference in means for gender and worksites. Cohen's formula generated effect size statistics $(0.25 \times$ (WS1 $(\mathrm{M} 3 S D+\mathrm{M} 4 S D)+\mathrm{WS} 2(\mathrm{M} 1 S D+\mathrm{M} 4 S D)))(15)$. The aforementioned measurement periods were chosen as they were unaffected by initial reactivity from wearing the pedometers. Confidence intervals were generated by using the Cohen effect size as a Pearson correlation value and setting $90 \%$ confidence limits. Paired $t$-statistics were used to ascertain effect differences between the worksites, and unequal variance $t$-statistics examined potential disparities between genders. Within-subject variations and interaction effects were generated using mixed modelling. Results were 
reported as three-day step values, and means \pm standard deviations were reported as whole numbers.

\section{Results}

Pedometer counts were grouped by gender and worksite to understand the physical activity contribution of total pedometer values (TPV), and work pedometer values (WPV) over the three days (Figure 1). Employees at both worksites accumulated $60 \%$ of physical activity in the workplace. 

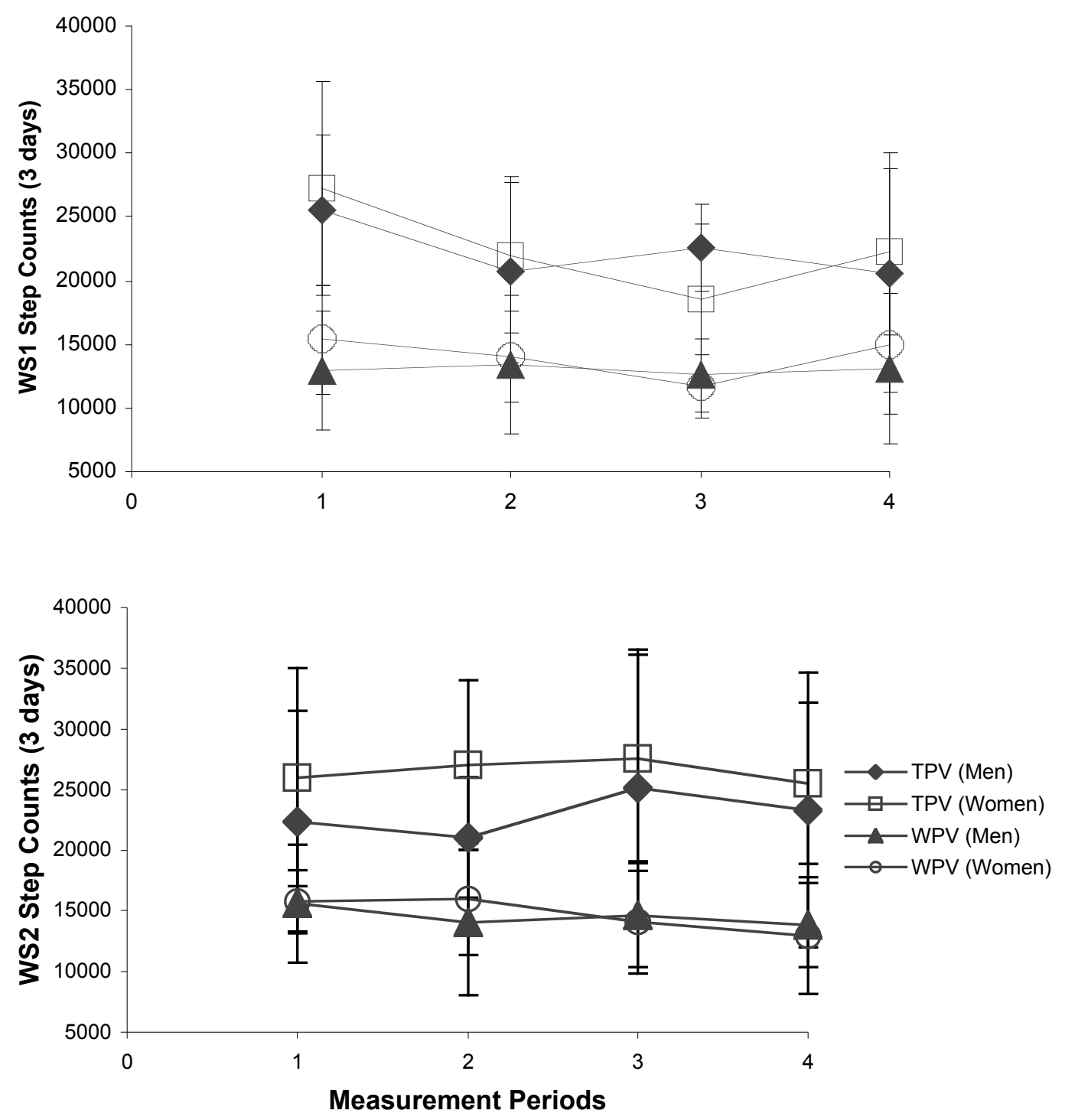

Figure 1: Mean pedometer step counts, defined by worksites, gender, total (TPV) and work (WPV) pedometer values for three days, over four measurement periods.I

Table 1 indicates step changes when the posters appeared. In the case of WS2, poster visibility slightly increased step counts. The posters' appearance was related to small step decreases in all other cases. Withinsubject variability was relatively large $(\mathrm{WPV}=35 \% ; 90 \% \mathrm{Cl}=31-41, \mathrm{TPA}=27 \%$; $90 \% \mathrm{Cl}=23-31)$. 
Table 1: Step changes, Cohen effect sizes and $t$-statistics derived from the appearance of posters in the worksite.

\begin{tabular}{|c|c|c|c|c|c|c|}
\hline & & $\begin{array}{l}\text { Step change } \\
\text { when posters } \\
\text { are visible }\end{array}$ & $\begin{array}{l}\text { Cohen } \\
\text { Effect }\end{array}$ & $\mathrm{Cl}(90 \%)$ & $\begin{array}{l}\text { Probability of } \\
\text { effect being } \\
\text { clinically harmful }\end{array}$ & t-statistic \\
\hline \multirow[t]{2}{*}{$\begin{array}{l}\text { WS1 } \\
(\mathrm{N}=24)\end{array}$} & WPV & -1899 & -0.45 & $\begin{array}{c}-0.69,- \\
0.13\end{array}$ & $96 \%$ & \\
\hline & TPV & -5338 & -0.79 & $\begin{array}{c}-0.89,- \\
0.61\end{array}$ & $100 \%$ & \\
\hline $\begin{array}{l}\text { WS2 } \\
(\mathrm{N}=22)\end{array}$ & $\begin{array}{l}\text { WPV } \\
\text { TPV }\end{array}$ & $\begin{array}{c}163 \\
1616 \\
\end{array}$ & $\begin{array}{l}0.04 \\
0.18 \\
\end{array}$ & $\begin{array}{l}-0.33,0.39 \\
-0.19,0.51\end{array}$ & $\begin{array}{l}27 \% \\
11 \%\end{array}$ & \\
\hline $\begin{array}{l}\text { Total } \\
\text { WS }\end{array}$ & $\begin{array}{l}\text { WPV } \\
\text { TPV }\end{array}$ & $\begin{array}{c}-868 \\
-1861 \\
\end{array}$ & $\begin{array}{l}-0.21 \\
-0.24 \\
\end{array}$ & $\begin{array}{l}-0.43,0.04 \\
-0.46,0.01\end{array}$ & $\begin{array}{l}77 \% \\
83 \% \\
\end{array}$ & $\begin{array}{l}0.65 \\
0.27\end{array}$ \\
\hline $\begin{array}{l}\text { Men } \\
(\mathrm{N}=27)\end{array}$ & $\begin{array}{l}\text { WPV } \\
\text { TPV }\end{array}$ & $\begin{array}{c}-268 \\
-1195\end{array}$ & $\begin{array}{l}-0.07 \\
-0.17\end{array}$ & $\begin{array}{l}-0.38,0.26 \\
-0.47,0.16\end{array}$ & $\begin{array}{l}44 \% \\
64 \%\end{array}$ & \\
\hline $\begin{array}{l}\text { Women } \\
(\mathrm{N}=19)\end{array}$ & $\begin{array}{l}\text { WPV } \\
\text { TPV }\end{array}$ & $\begin{array}{l}-1625 \\
-2942 \\
\end{array}$ & $\begin{array}{l}-0.37 \\
-0.35 \\
\end{array}$ & $\begin{array}{l}-0.66,0.02 \\
-0.65,0.05\end{array}$ & $\begin{array}{l}88 \% \\
86 \% \\
\end{array}$ & \\
\hline \multirow{2}{*}{$\begin{array}{l}\text { Total } \\
\text { Gender }\end{array}$} & WPV & -868 & -0.21 & $-0.43,0.04$ & $77 \%$ & 0.47 \\
\hline & TPV & -1861 & -0.24 & $-0.46,0.01$ & $83 \%$ & 0.66 \\
\hline
\end{tabular}

Effect sizes for step count changes ranged from trivial (0.04) to moderate (0.79) effects. The majority of reported Cohen effect sizes were small, negative changes. Gender and worksite changes for the posters' appearance were similar as indicated by the small $t$-statistics. Mixed modelling identified that no intervention carry-over effect was evident in the cross over design $(\mathrm{WPV}=-0.4 \%, \mathrm{TPV}=-0.02 \%)$.

\section{Discussion}

This is the first study to examine poster efficacy using the same participants repeatedly with an objective measure of physical activity. Our findings indicate the posters do not increase pedometer-measured step counts in this sample of professional employees. In contrast, previous research using head counts as the primary evaluation measure reported small absolute increases in stair use $(8,11,13)$.

A potential reason for the present worksite differentiation is stairwell access. WS1's stairwell is only swipe card accessible (all participants had 
cards), enclosed with artificial lighting, and located a distance from the lifts. Conversely, WS2 has an open stairwell, is lit naturally, and is adjacent to the lifts. From an urban design viewpoint, stairwells should always be visible from a building's main point of entry, preferably with the lifts hidden to the side.

Previous research found that women were more likely to take the stairs once an intervention occurs $(6,10,13)$. This not the case in the present study, with women more likely reducing their step counts in comparison to men. A possible explanation for this finding is that the smaller sample of women $(\mathrm{N}=19)$ is more sensitive to change.

There are several limitations to the present study. No self-report data were obtained. Therefore barriers for stair use in this sample remain unknown. Many factors could have been responsible for the step decrease, including the size, placement (14), message content (13), or feelings of guilt (6) associated with the posters. This sample is unrepresentative of all working adults and can only be applied to similar occupations. Finally, the small sample size limits the efficacy of the confidence intervals to detect changes in activity levels. An adequate sample size would be 64 employees at each site.

\section{Conclusions}

On the basis of our findings, we believe that at least the posters we used, are not effective for increasing physical activity levels in professional employees. However, these results should be treated as preliminary. Nothing is known about the effect of posters for different occupational categories, other types of posters, or when posters are integrated with a 
broader multi-strategy intervention. Further research is needed to validate the findings reported in the present study.

\section{Acknowledgments}

The authors' wish to thank Sport and Recreation New Zealand (SPARC) for funding the pedometers in the study, and the New Zealand National Heart Foundation for providing the posters. 


\section{References}

1. Department of Health, Physical Activity, Health Improvement and Promotion. At least five a week: Evidence on the impact of physical activity and its relationship to health. A report from the Chief Medical Officer. 2004. Department of Health, London.

2. Hardman, A. Issues of fractionization of exercise: Long versus short bouts. Medicine and Science in Sports and Exercise 2001;(33):S421-S427.

3. Bauman, A., Bellew, B., Vita, P., Brown, W., \& Owen, N. Getting Australia active: Towards better practice for the promotion of physical activity. 2002. National Public Health Partnership, Melbourne.

4. Boreham, C., Wallace, W., \& Nevill, A. Training effects of accumulated daily stair-climbing exercise in previously sedentary young women. Preventive Medicine 2000;(30)277-281.

5. Kerr, J., Eves, F., \& Carroll, D. Can posters prompt stair use in a worksite environment? American Journal of Public Health 2001;(91):1192-1193.

6. Kerr, J., Eves, F., \& Carroll, D. Encouraging stair use: Stair-riser banners are better than posters. Journal of Occupational Health 2001;(43):205-207.

7. Titze, S., Martin, B., Seiler, R., \& Marti, B. A worksite intervention module encouraging the use of stairs: Results and evaluation issues. Preventive Medicine 2001;(46):13-19.

8. Russell, W., Dzewaltowski, D., \& Ryan, G. The effectiveness of point of decision prompts in deterring sedentary behavior. American Journal of Health Promotion 1999;(13):257-259.

9. Coleman, K., \& Gonzalez, E. Promoting stair use in a US-Mexico border community. American Journal of Health Promotion 2001;(91):2007-2015.

10. Boutelle, K., Jeffrey, R., Murray, D., \& Schmitz, K. Using signs, artwork, and music to promote stair use in a public building. American Journal of Public Health 2001;(91):2004-2010.

11. Marshall, A., Bauman, A., Patch, C., Wilson, J., \& Chen, J. Can motivational signs prompt increases in incidental physical activity in an Australian health-care facility? Health Education Research 2002;(17):743-749.

12. Blamey, A., Mutrie, N., \& Aitchison, T. Health promotion by encouraged use of stairs. British Medical Journal 1995;(311):289-290. 
13. Anderson, R., Franckowiak, S., Snyder, J., Bartlett, S., \& Fontaine, K. Can inexpensive signs encourage the use of stairs? Results from a community intervention. Annals of Internal Medicine 1998;(129)363-369.

14. Kerr, J., Eves, F., \& Carroll, D. The influence of poster prompts on stair use: The effects of setting, poster size, and content. British Journal of Health Psychology 2001;(6):367-405.

15. Hopkins, W. Calculating likely (confidence) limits and likelihoods for true values. In: A new view of statistics. 2003. Sportsci.org: Internet Society for sports sciences.

16. Lind, C. (19 February 2003). Personal communication. H. Badland (Ed.). Auckland. 